\title{
Transmissibilty-based operational modal analysis for flight flutter testing using exogenous inputs
}

\author{
Christof Devriendt, Tim De Troyer*, Gert De Sitter and Patrick Guillaume \\ Acoustic and Vibration Research Group, Vrije Universiteit Brussel, Brussel, Belgium
}

\begin{abstract}
During the recent years several new tools have been introduced by the Vrije Universiteit Brussel in the field of Operational Modal Analysis (OMA) such as the transmissibility based approach and the the frequency-domain OMAX concept. One advantage of the transmissibility based approach is that the ambient forces may be coloured (non-white), if they are fully correlated. The main advantage of the OMAX concept is the fact that it combines the advantages of Operational and Experimental Modal Analysis: ambient (unknown) forces as well as artificial (known) forces are processed simultaneously resulting in improved modal parameters. In this paper, the transmissibility based output-only approach is combined with the input/output OMAX concept. This results in a new methodology in the field of operational modal analysis allowing the estimation of (scaled) modal parameters in the presence of arbitrary ambient (unknown) forces and artificial (known) forces.
\end{abstract}

Keywords: Operational modal analysis, transmissibility, flutter

\section{Introduction}

The idea to combine experimental and operational vibration data emerged from flight flutter testing. In those tests [1], the aircraft's structure is excited by an applied force and by the atmospheric turbulence. Another typical example is the modal analysis of bridges: the structure is excited by ambient forces (wind, traffic) and by applied forces [2]. In classical Experimental Modal Analysis [3,4], the ambient excitation is considered as a disturbing noise. In Operational Modal Analysis however [5-8], one only uses the response of the system caused by the unknown ambient forces. Modal parameter estimators in the OMAX framework consider both the deterministic contribution caused by the applied forces and the stochastic contribution caused by the unmeasurable forces [9-11]. Both contributions contain useful information on the system.

As in classical frequency-domain EMA and OMA, the input data can be (averaged) spectral functions (i.e. frequency response function FRF and auto- and crosspower spectrum XP), or the raw input and output (I/O) Fourier coefficients. The single-reference common-denominator model, the polyreference matrix-fraction description, and the state-space model can be used [3]. The use of the frequency domain allows easy pre-filtering of the data and fast yet accurate algorithms to estimate the modal parameters [12]. The FRF and XP are constructed from the measured signals using the FFT and correlation techniques.

In this paper, we investigate the use of transmissibility functions as defined in [13,14]. A transmissibility (TM) is the ratio between two responses, and thus it can be calculated without knowing what forces cause these responses. Recently an operational modal analysis approach using transmissibility functions as primary data has been developped [13]. The research was mainly driven by finding solutions for some disadvantages of current OMA

*Corresponding author: Tim De Troyer, Erasmushogeschool Brussel, Department of Industrial Sciences and Technology, Nijverheidskaai 170, B-1070, Brussels, Belgiume. E-mail: cdevrien@ vub.ac.be. 
techniques using power spectra as primary data. One disadvantage of the current OMA techniques is that the modal parameters are sometimes wrongly identified when the forces applied to the structure are non-white noise sequences. The main advantage of TM is that the ambient forces may be coloured (non-white), if they are fully correlated [15]. This is e.g. true if the ambient force is dominated by a discrete source such as an engine or a gearbox, or even turbulence acting on an aircraft. This property can also be used to advantage in an OMAX framework, by combining FRFs with TMs instead of XPs.

The requirement for the transmissibility approach is that the technique does need changing loading conditions. The changing loading conditions can either be obtained by a change in the ambient forces, e.g. changing direction or amplitude of wind or by adding an artificial force. This latter would benefit from an OMAX-framework.

OMAX is really advantageous when some modes are not (well) excited by the applied force. This can be because it is difficult to inject sufficient energy at specific frequencies (e.g. bridges). It can also be that the force is applied in a node of a mode. In this case, an operational (broadband) excitation can reveal such a mode.

In Section 2, we introduce the concept of OMAX in a slightly more formal way. In Section 3, the use of transmissibility functions is explained, both for OMA and OMAX applications. The proposed approach is tested on simulations in Section 4 and applied to flight flutter testing in Section 5. Finally the conclusions are drawn.

\section{The omax idea}

In general, a mechanical structure under test is also excited by unmeasurable forces. $\operatorname{Say} \mathbf{f}(t)$ is the vector of $N_{i}$ applied forces, $\mathbf{y}(t)$ the vector of $N_{o}$ measured responses, and $\mathbf{e}(t)$ the vector of $N_{e}$ unknown excitations. A classical test is visualised in Fig. 1.

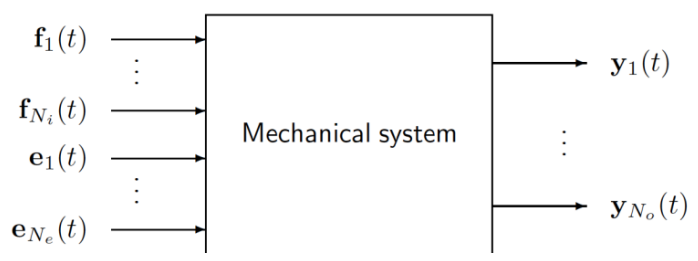

Fig. 1. Responses ( $\mathbf{y}(t))$ of a mechanical system due to measured $(\mathbf{f}(t))$ and unmeasured $(\mathbf{e}(t))$ excitations.

The classical EMA approach tries to reduce this noise influence by averaging to estimate a nonparametric FRF. The FRF $\mathbf{H}\left(\omega_{k}\right)$ relates the Fourier transform of the force $\mathbf{F}\left(\omega_{k}\right)$ and response $\mathbf{Y}\left(\omega_{k}\right)$ as:

$$
\mathbf{Y}\left(\omega_{k}\right)=\mathbf{H}\left(\omega_{k}\right) \mathbf{F}\left(\omega_{k}\right)
$$

with $\omega_{k}$ the discrete angular frequency at index $k$. In operational conditions however, $\mathbf{f}(t)=\mathbf{0}$ and the auto- and crosspower spectrum of the responses is related to the unknown operational excitation $\mathbf{e}(t)$ as:

$$
\mathbf{S}_{\mathbf{Y Y}}\left(\omega_{k}\right)=\mathbf{H}\left(\omega_{k}\right) \mathbf{S}_{\mathbf{E E}}\left(\omega_{k}\right) \mathbf{H}^{H}\left(\omega_{k}\right)
$$

with $\mathbf{S}_{\mathbf{E E}}\left(\omega_{k}\right)$ the auto- and crosspower spectrum of the unknown forces, $\mathbf{E}\left(\omega_{k}\right)$ is the Fourier transform of $\mathbf{e}(t)$. It is generally assumed that $\mathbf{S}_{\mathbf{E E}}\left(\omega_{k}\right)$ is a constant matrix over the frequencies.

If both known and unknown forces excite the structure under test, then the spectrum of the responses is found by extending Eq. (1) as

$$
\mathbf{Y}\left(\omega_{k}\right)=\mathbf{H}\left(\omega_{k}\right) \mathbf{F}\left(\omega_{k}\right)+\mathbf{G}\left(\omega_{k}\right) \mathbf{E}\left(\omega_{k}\right)
$$

with $\mathbf{G}\left(\omega_{k}\right)$ that part of the FRF that describes the relation between the (unknown) input locations and the measured output stations. Both $\mathbf{H}\left(\omega_{k}\right)$ and $\mathbf{G}\left(\omega_{k}\right)$ depend only on the inherent $\mathbf{M}, \mathbf{C}, \mathbf{K}$ structure of the system under test. 
From modal analysis theory [3], we know that the poles are global properties of the system. Practically, this means that the resonant frequencies and damping ratios are independent of the location of the force and response measurement. If this principle is applied to the system in Fig. 1, it follows that both $\mathbf{H}\left(\omega_{k}\right)$ and $\mathbf{G}\left(\omega_{k}\right)$ will have the same poles, and thus the same denominator. The modal participation factors will differ, since the unknown force location will differ in general from the applied excitation. The goal of the OMAX framework is to improve the accuracy of the estimates of the poles, based on the principle that $\mathbf{H}\left(\omega_{k}\right)$ and $\mathbf{G}\left(\omega_{k}\right)$ have the same denominator. This idea can be implemented in two ways:

- as the estimation of a combined parametric system model (plant model) $\mathbf{H}\left(\omega_{k}\right)$ and a noise model $\mathbf{G}\left(\omega_{k}\right)$ using $\mathbf{Y}\left(\omega_{k}\right)$ and $\mathbf{F}\left(\omega_{k}\right)$ as input data,

- or as the combined parametric estimation of the (nonparametric) FRF and the response crosspower spectra $(\mathrm{XP})$.

The former implementation is similar to the prediction error minimisation of an auto-regressive moving-average with exogenous inputs (ARMAX) process in the time domain [17]. The latter implementation is inspired by multivariable parameter estimation: next to the multiple FRF measurements, also the XP are included and identified simultaneously.

Inspired by Eq. (3), a common-denominator model can be formulated as

$$
\mathbf{Y}\left(\omega_{k}\right)=\frac{\mathrm{N}(\omega, \boldsymbol{\theta})}{\mathrm{D}(\omega, \boldsymbol{\theta})} \mathbf{F}\left(\omega_{k}\right)+\frac{\mathrm{C}(\omega, \boldsymbol{\theta})}{\mathrm{D}(\omega, \boldsymbol{\theta})} \mathbf{E}\left(\omega_{k}\right)
$$

with $\mathrm{N}\left(\Omega_{k}, \boldsymbol{\theta}\right)$ the FRF model numerator $\left(N_{o} \times N_{i}\right), \mathrm{C}\left(\Omega_{k}, \boldsymbol{\theta}\right)$ the noise model numerator $\left(N_{o} \times N_{e}\right)$, and $\mathrm{D}\left(\Omega_{k}, \boldsymbol{\theta}\right)$ the scalar denominator, common for the FRF model $\boldsymbol{H}\left(\Omega_{k}, \boldsymbol{\theta}\right)$ and the noise model $\boldsymbol{G}\left(\Omega_{k}, \boldsymbol{\theta}\right)$.

In classical EMA, the FRF is the data that is curve-fitted, while in OMA the power spectra are the source data. A combined EMA-OMA approach is thus the combined estimation of FRF and XP.

The FRF and the XP both have the same system poles, as can be seen from Eq. (2). The only difference is that the XP have a four-quadrant symmetry, i.e. all poles $\lambda_{r}$ of the FRF appear also as unstable poles $-\lambda_{r}$ in the XP. One way to circumvent this is by calculating the positive power spectra $\left(\mathrm{XP}^{+}\right)$[18]. Both the FRF and the $\mathrm{XP}^{+}$can be curve-fitted simultaneously, e.g. using a common-denominator model (cd) or a right matrix-fraction description (RMFD). The resulting error equation is given by

$$
\begin{aligned}
& \varepsilon_{c d}\left(\Omega_{k}, \boldsymbol{\theta}\right)=\left[\begin{array}{ll}
\boldsymbol{H}\left(\omega_{k}\right) & \mathbf{S}_{\mathbf{Y} \mathbf{Y}}^{+}\left(\omega_{k}\right)
\end{array}\right]-\frac{\mathrm{N}(\omega, \boldsymbol{\theta})}{\mathrm{D}(\omega, \boldsymbol{\theta})} \\
& \varepsilon_{R M F D}\left(\Omega_{k}, \boldsymbol{\theta}\right)=\left[\begin{array}{ll}
\boldsymbol{H}\left(\omega_{k}\right) & \mathbf{S}_{\mathbf{Y Y}}^{+}\left(\omega_{k}\right)
\end{array}\right]-\mathrm{N}_{R}(\omega, \boldsymbol{\theta}) \mathrm{D}_{R}^{-1}(\omega, \boldsymbol{\theta})
\end{aligned}
$$

where $\mathrm{N}\left(\Omega_{k}, \boldsymbol{\theta}\right)$ and $\left.\mathrm{N}_{R}\left(\Omega_{k}, \boldsymbol{\theta}\right)\right)$ are now $N_{o} \times\left(N_{i}+N_{\text {ref }}\right)$ polynomial matrices with $N_{\text {ref }}$ the number of output responses used to calculate the power spectrum $\left(\mathbf{S}_{\mathbf{Y Y}}^{+}\right.$is $N_{o} \times N_{\text {ref }}$ ). Note that, for the RMFD, the size of the denominator matrix must be increased to $\left(N_{i}+N_{\text {ref }}\right) \times\left(N_{i}+N_{\text {ref }}\right)$. A left matrix-fraction description is also possible, but since $N_{o}$ is in general much larger than $N_{i}$ in modal analysis, we prefer to use a RMFD to limit the calculation time. Since Eq. (5) is equivalent to the error equation of the classic LSCF estimator, the LSCF algorithms can be used without any modification. This means that all FRF-based modal parameter estimators can be

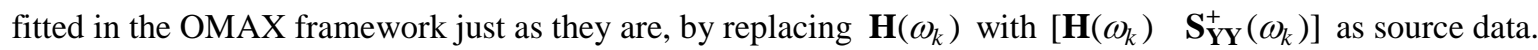

\section{Oma and omax using transmissibility functions}

The (single-reference) transmissibility function is here defined as the ratio between two response spectra $i$ and $j$ : 


$$
T_{i j}^{A}=\frac{Y_{i}\left(\omega_{k}\right)}{Y_{j}\left(\omega_{k}\right)}
$$

while the structure is subjected to a certain loading condition $A$. These functions can be estimated in a non-parametric way comparable to power spectra, e.g. by dividing data into blocks and averaging.

The use of transmissibility functions as primary data for output-only modal analysis has only recently been considered and was introduced for the first time in Devriendtmssp. It was shown that transmissibility functions could be post-processed into suitable primary data for existig system identification techniques [10] in the field of operational modal analysis [5]. The key idea behind the technique is that a set of transmissibility functions becomes independent of the applied forces exactly in the system poles and that their value in the system poles is related to the mode shape values. The limit value of the transmissibility function for the Laplace variable $s$ going to the system's poles, $\lambda_{m}$, converges to

$$
\lim _{s \rightarrow \lambda_{m}} T_{i j}^{A}(s)=\frac{\phi_{i m}}{\phi_{j m}}
$$

and becomes independent of the (unknown) forces. This fact that the transmissibility function becomes independent of the locations and the amplitudes of the applied forces exactly at the system poles can now be used to find the system poles that were canceled out by taking the ratio of two responses.

Take for example the subtraction of two transmissibility functions with the same output DOFs, $(i, j)$, but with different loading conditions, $(A, B)$, e.g. different locations or different amplitudes of the unknown forces, $F_{k}(s)$. This satisfies

$$
\lim _{s \rightarrow \lambda_{m}}\left(T_{i j}^{A}(s)-T_{i j}^{B}(s)\right)=\frac{\phi_{i m}}{\phi_{j m}}-\frac{\phi_{i m}}{\phi_{j m}}=0
$$

This means that the system's poles, $\lambda_{m}$, are zeroes of the rational function $\Delta T_{i j}^{A B}(s)=T_{i j}^{A}(s)-T_{i j}^{B}(s)$, and, consequently, poles of its inverse, i.e.

$$
\Delta^{-1} T_{i j}^{A B}(s)=\frac{1}{\Delta T_{i j}^{A B}(s)}=\frac{1}{T_{i j}^{A}(s)-T_{i j}^{B}(s)}
$$

The above elaborated theoretical results show that it is possible, by using transmissibility functions, to obtain a rational function $\Delta^{-1} T_{i j}^{A B}(s)$, with poles equal to the system's poles. Note that the rational function $\Delta^{-1} T_{i j}^{A B}(s)$ can be rewritten as (for the simple input cases in DOF $k$ and $l$ )

$$
\Delta^{-1} T_{i j}^{k l}(s)=\frac{1}{\frac{H_{i k}(s)}{H_{j k}(s)}-\frac{H_{i l}(s)}{H_{j l}(s)}}=\frac{H_{j k}(s) H_{j l}(s)}{H_{i k}(s) H_{j l}(s)-H_{i l}(s) H_{j k}(s)}=\frac{N_{j k}(s) N_{j l}(s)}{N_{i k}(s) N_{j l}(s)-N_{i l}(s) N_{j k}(s)}
$$

and so, the order of the polynomial, $N_{i k}(s) N_{j l}(s)-N_{i l}(s) N_{j k}(s)$, can exceed the order of the commondenominator polynomial, $D(s)$. This means that $\Delta^{-1} T_{i j}^{A B}(s)$ can contain more poles than the system's poles only. Hence, in general, only a subset of the poles of $\Delta^{-1} T_{i j}^{A B}(s)$ will correspond to the real system's poles. To select the correct system poles, a singular value decomposition can, for instance, be used [14]. A very important remark is that the rational transfer function $\Delta^{-1} T_{i j}^{A B}$ is not unique: several different functions can be defined starting from transmissibility functions with poles equal to the the system poles. We could easily extend this function by combining all the measured transmissibility functions in one single rational function that still has roots equal to the system's poles. The subtraction of the summation of all measured transmissibility functions under one loading condition $k, T_{i j}^{k}(s)$ for $i=1 \ldots N_{o}$ with $N_{o}$ the number of output DOF, and the summation of all measured 
transmissibility functions under a reference loading condition $r e f, T_{i j}^{r e f}(s)$, and this summed over all loading conditions $k, k=1 \ldots N_{l}$ with $N_{l}$ the number of loading conditions, still satisfies

$$
\lim _{s \rightarrow \lambda_{m}} \sum_{k=1}^{N_{l}}\left(\sum_{i=1}^{N_{o}} T_{i j}^{k}(s)-\sum_{i=1}^{N_{o}} T_{i j}^{r e f}(s)\right)=0
$$

This means that the system's poles, $\lambda_{m}$ are the poles of the rational function

$$
\Delta^{-1} T(s)=\left(\sum_{k=1}^{N_{l}}\left(\sum_{i=1}^{N_{o}} T_{i j}^{k}(s)-\sum_{i=1}^{N_{o}} T_{i j}^{r e f}(s)\right)\right)^{-1}
$$

Therefore by applying directly the SISO-least squares frequency domain estimator to this rational function, one could again obtain the system's poles. A pseudo FRF can then be constructed using Eq. (12) as denominator, and transmissibility functions as numerator:

$$
\mathbf{H}_{T}\left(\omega_{k}\right)=\frac{\left[\begin{array}{ccc}
T_{1 j}^{1}\left(\omega_{k}\right) & \cdots & T_{1 j}^{N_{l}} \\
\vdots & \ddots & \vdots \\
T_{N_{o} j}^{1}\left(\omega_{k}\right) & \cdots & T_{N_{o} j}^{N_{l}} \\
& &
\end{array}\right]}{\Delta T\left(\omega_{k}\right)}
$$

From this matrix we can obtain both the poles and the unscaled mode shape vectors from transmissibility measurement in a single identification step by applying existing frequency domain estimators [16]. This pseudo FRF will be used in this paper, to be combined with the classical force-response FRF. It will be shown that all FRF-based modal parameter estimators can also be fitted in the OMAX framework just as they are by using $\left[\begin{array}{ll}\mathbf{H}\left(\omega_{k}\right) & \mathbf{H}_{\mathbf{T}}\left(\omega_{k}\right)\end{array}\right]$ as source data.

\section{Simulations: Flight flutter testing}

In this paper simulations will be used to give a proof of concept on how transmissibility measurements could be used in the field of flight flutter testing in both an OMA as OMAX framework. We will also compare the results obtained from applying the LSCF estimator to FRFs, Powers Spectra and Transmissibility-based functions. The simulations make use of a modal model that is extracted from a real ground vibration test (GVT) of an aircraft, which geometry shown in Fig. 2. Only 38 output degrees-of-freedom (DOFs) of the originally 543 measured DOFs are used to perform the simulations.
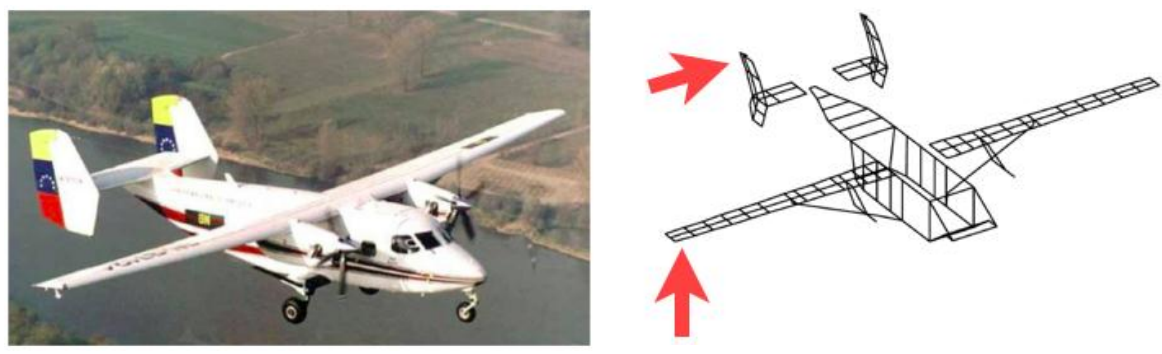

Fig. 2. Tested Aircraft. 
The simulations were done directly in the frequency domain (no leakage is considered) with a modal model based on the first six modes only, visualized in Fig. 3. The frequency band is $1.5 \mathrm{~Hz}-10 \mathrm{~Hz}$ and 545 frequency lines have been considered resulting in a frequency resolution of $0.0156 \mathrm{~Hz}$.
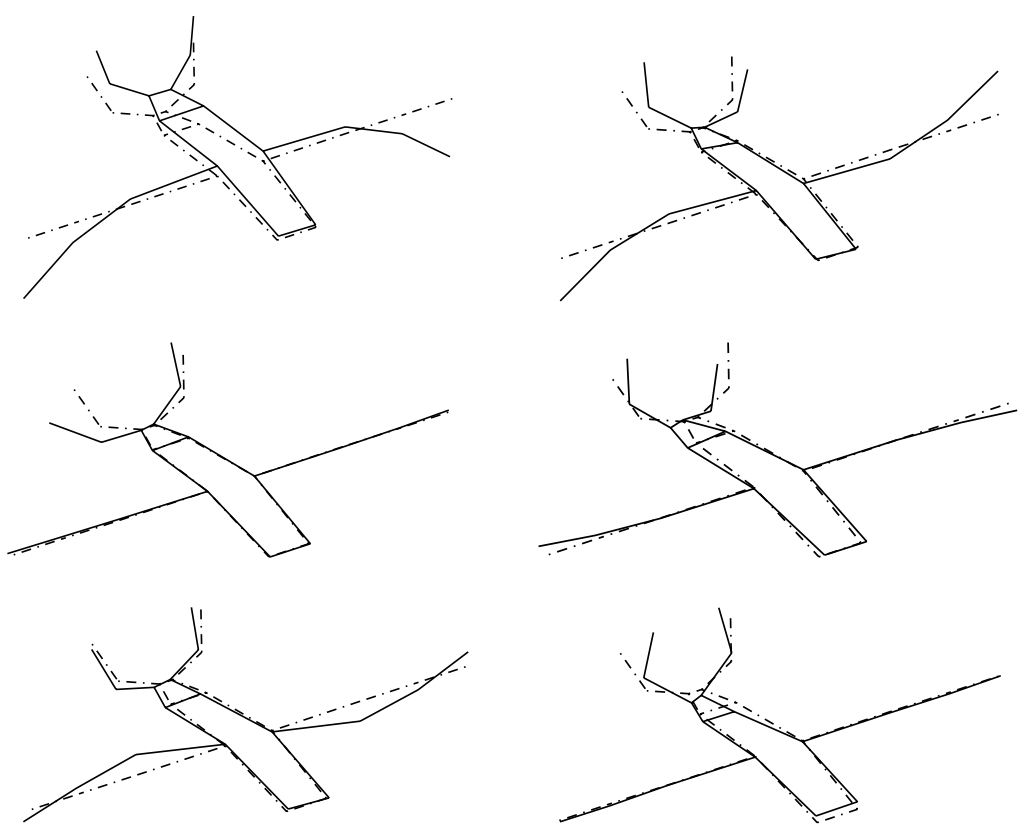

Fig. 3. Mode shapes of airplane.

In order to apply the transmissibility approach two different loading conditions where applied. In the first simulation, one random white noise force $f(t)$ is applied to the end of the left wing in vertical direction. This force will be considered as a known force in order to obtain the FRF's for the input-output estimation. In the second simulation, an extra random white noise force $f(t)$ is applied to the top-end of the left tail-wing in horizontal direction.

For the transmissibility approach we will use following pseudo transfer matrix.

$$
\mathbf{H}_{T}\left(\omega_{k}\right)=\frac{\left[\begin{array}{cc}
T_{11}\left(\omega_{k}\right) & T_{12}\left(\omega_{k}\right) \\
\vdots & \vdots \\
T_{N_{o} 1}\left(\omega_{k}\right) & T_{N_{o} 2}\left(\omega_{k}\right)
\end{array}\right]}{\Delta T\left(\omega_{k}\right)}
$$

with $N_{o}=38$ and with the reference response taken on the end of the left wing in vertical direction. With $\Delta T\left(\omega_{k}\right)$ obtained using all 38 responses and the first loading condition as reference loading condition. The exact elements of this pseudo transfer matrix $H_{T}\left(\omega_{k}\right)$ are shown in Fig. 4 (only the first column is shown). One can observe that each element of this pseudo transfer matrix $H_{T}\left(\omega_{k}\right)$ has peaks at frequencies that coincide with the 6 resonance frequencies of the simulated modes of the airplane.

To simulate the influence of the atmospheric turbulence, correlated white noise sources are considered on the nose, wings and tail of the aircraft. Simulations were carried out for one level of atmospheric turbulence in all 38 DOFS: $10 \%$ of the applied random noise force $f(t)$. For each simulation 4 measurements blocks where taken in order to average. We can now apply the least square frequency domain estimator directly on the measured transmissibility based pseudo transfer matrix $H_{T}\left(\omega_{k}\right)$, the power spectra and the FRFs. The data-sets used for all identification 


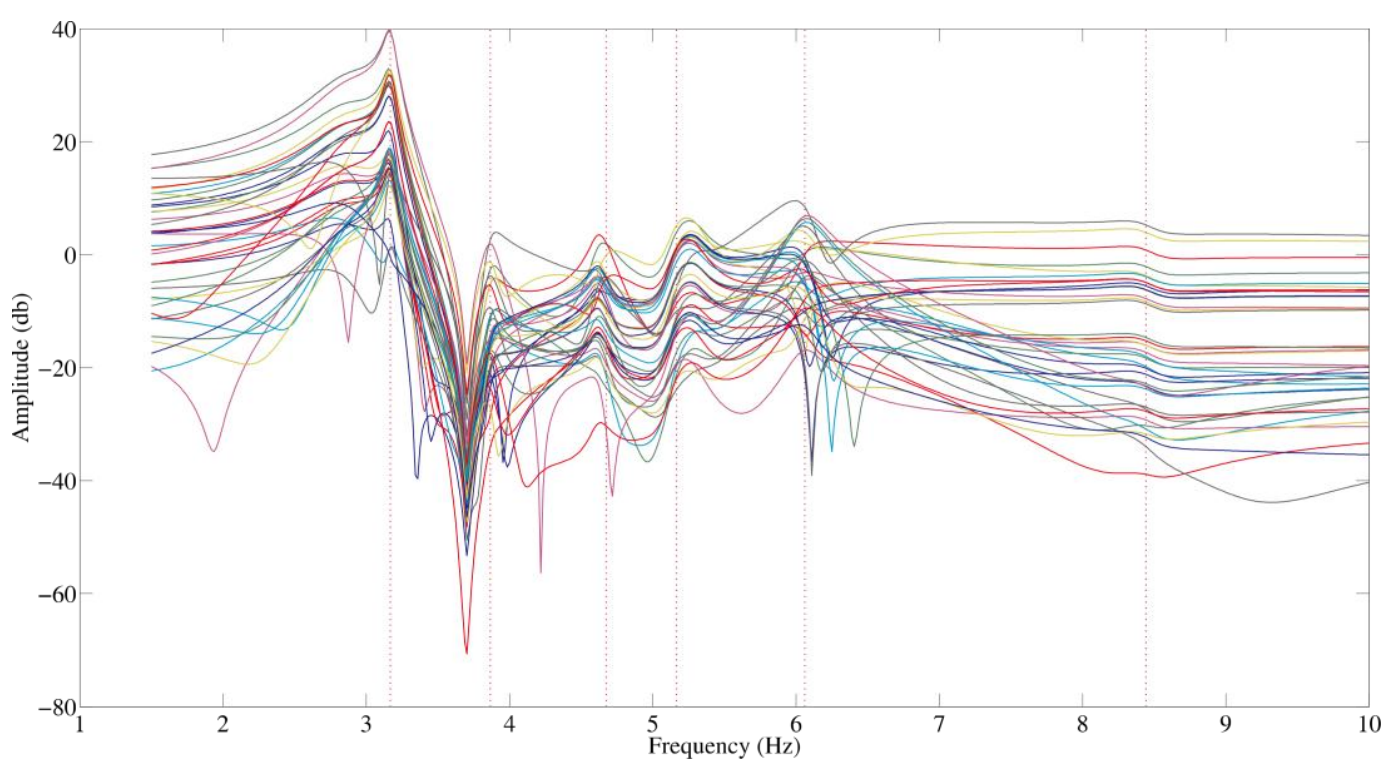

Fig. 4. Exact elements of the pseudo transfer matrix $H_{T}\left(\omega_{k}\right)$.

approaches consist of the 2 different loading conditions where both the atmospheric turbulence and the applied random white noise force are present. The stabilization diagrams for a model order of 64 are illustrated in Fig. 5.

In the stabilization diagram algorithm used in this paper we evaluate for every pole, the distance to the nearest pole calculated with the previous model order and we plot:

- a red star, if distance is smaller than $1 \%$,

- a blue cross, if only frequency variation is smaller than $1 \%$,

- a black circle, if only damping variation is smaller than $5 \%$,

- a black star, if neither the pole, nor the frequency, nor the damping value stabilises

We can observe that for this example clear stabilization diagrams are found using FRFs. Six stable poles are found in frequency band of interest. The third, fourth and sixth are less clear since these are tail modes that are not well excited by the applied force at the tip of the left wing. When using the power spectra only the first mode results in a clear stable pole. The transmissiblity-based approach results in better results than the power spectra. In this stabilization diagram several stable poles can easily be identified.

One of the most important parameters in flight flutter testing is the damping ratio. Therefore 10 runs are considered to check for systematic errors and the variability on the estimated damping ratio estimates of each mode for the 3 approaches. For each run 6 poles, those closest to the exact poles, were automatically selected for all 3 approaches, so no manual selection by a stabilization diagram was done. One can conclude that the results obtained from the FRFs and the transmissibility functions are mostly in good agreement with the exact value of the damping ratio, except for the third and fourth mode. For these modes the errors on the FRF approach are smaller than the transmissibility based approach. On the other hand the damping values estimated from the power spectra are underestimated for all runs and all modes. except for the first mode.

The above experiment shows that the use of transmissibility measurements for flight flutter testing might be interesting and is definitely worth to investigate in more detail. The authors are well aware that real experiments will be contaminated with a higher level of noise and issues such as leakage because of the short data records. In bart it was shown that the FRF method suffers from a bias on the damping ratios of the low frequency modes due to the leakage effects in the FRF estimates and is not recommended to use in the case of short time records. The same issues can be expected for transmissibility measurements. So called input-output driven estimators bart are proposed in this case. 

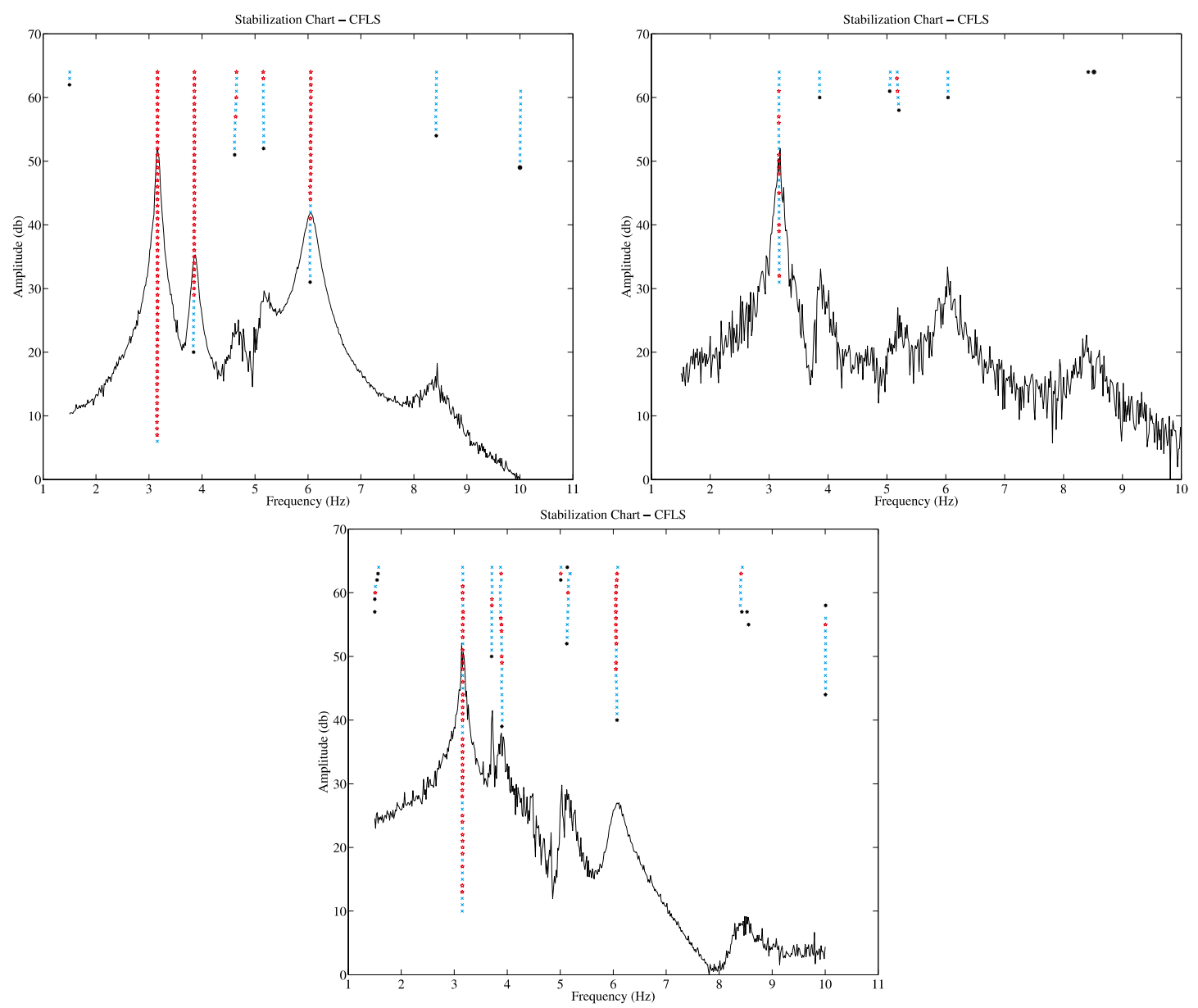

Fig. 5. Stabilization diagrams based on (top left) FRFs (top right) power spectra based OMA (bottom) Transmissibility based OMA.

The above experiments illustrated that the results obtained by transmissibility measurements coincide better with the results obtained from FRFs than the power spectra do. We can now apply the least square frequency domain estimator in an OMAX framework by combining the transmissibility based pseudo transfer matrix $H_{T}(\omega)$ with the FRF measurements and by combining the power spectra with the FRF measurments. The stabilization diagrams are illustrated in Fig. 7.

We can observe that the OMAX approach allows, by using a proper weighting, to improve the quality of the stabilization diagrams, Fig. 7, and the damping ratio estimates, Fig. 8, in comparison with the OMA approaches.

\section{Application to real flutter test data}

We have applied the OMAX procedure using power spectra (XP) and transmissibilities (TM) to real flight flutter test data. The test has been performed on a commercial aircraft equipped with fly-by-wire control. This primary flight control system was also used as excitation device: the electric force signal was superposed on the (auto)pilot's control signal and fed to the ailerons. The excitation signal was a sine-sweep that was sent to both ailerons, but with a phase lag of $\pi$ radians, resulting in an anti-symmetrical excitation. In this way both mainly the anti-symmetrical modes of the aircraft are excited. The 'symmetry' we mention here is with regard to the aircraft's plane of symmetry, i.e. the plane spanned by the top and the longitudinal axis. Loosely said, we observe an anti-symmetrical mode when one wing goes up and the other one goes down (e.g. the second mode in Fig. 3). Multiple accelerometers 
were used to capture the response of the aircraft, but we limited our OMAX test to four outputs. The sampling frequency was $256 \mathrm{~Hz}$. Figure 9 shows the force signal for the port aileron, and one response at the port wing tip. These measurements can be split up in two parts: in the first part, the aircraft is excited with the sine sweep (EMA); in the second part no extra excitation was applied but the response of the aircraft (due to e.g. turbulence and vibration from the engines) was still measured (OMA).
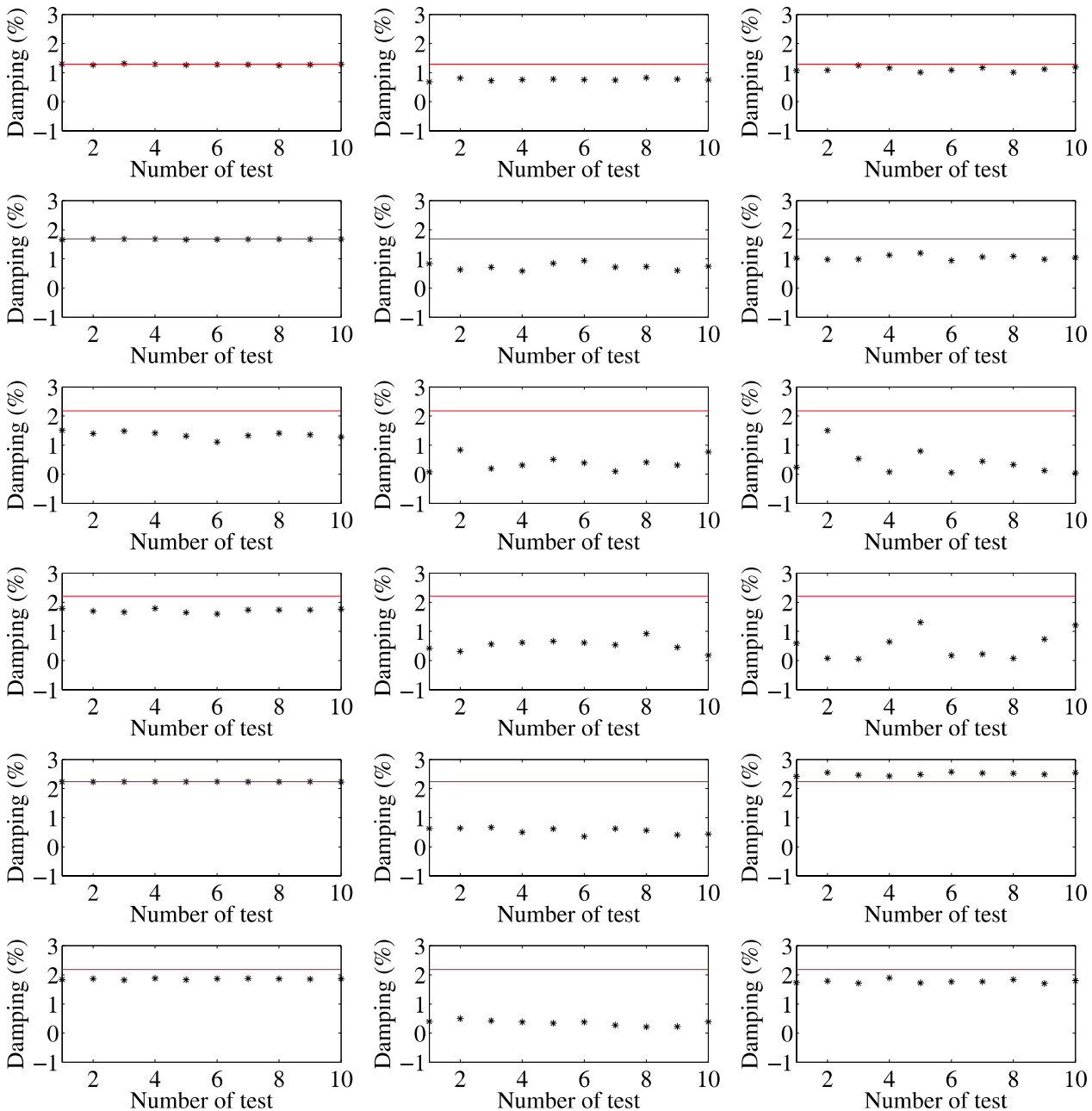

Fig. 6. Damping estimates based on (left) FRFs (middle) power spectra based OMA (right) Transmissibility based OMA.

We processed both parts separately to determine the FRF (from the first part) and the $\mathrm{XP}^{+}$(from the second part), since the stochastic part of the response was immediately available. Moreover, this data gives a nice example of how different loading conditions can be obtained in practice: responses were measured with the exciters on and off. The FRF is estimated using the $H_{1}$ approach with 7 averages and 50\% overlap. Each block has 4096 samples. A Hanning window is applied to the time data to prevent leakage errors. The FRF and the corresponding standard deviation for one output are shown in Fig. 10, left. This FRF is curve-fitted with a model order 32. This overmodelling is necessary to account for model errors and unmodelled dynamics. We used the maximum likelihood estimator and we allowed sufficient iterations for the MLE to converge by minimizing a cost function, as could be done in an after-flight analysis mle. In this way, we focus on the applicability of OMAX to flutter testing. Figure 10 shows a sample FRF with the MLE fit. Note that we have omitted the frequency scale in the figure as was requested by the aircraft's manufacturer. 

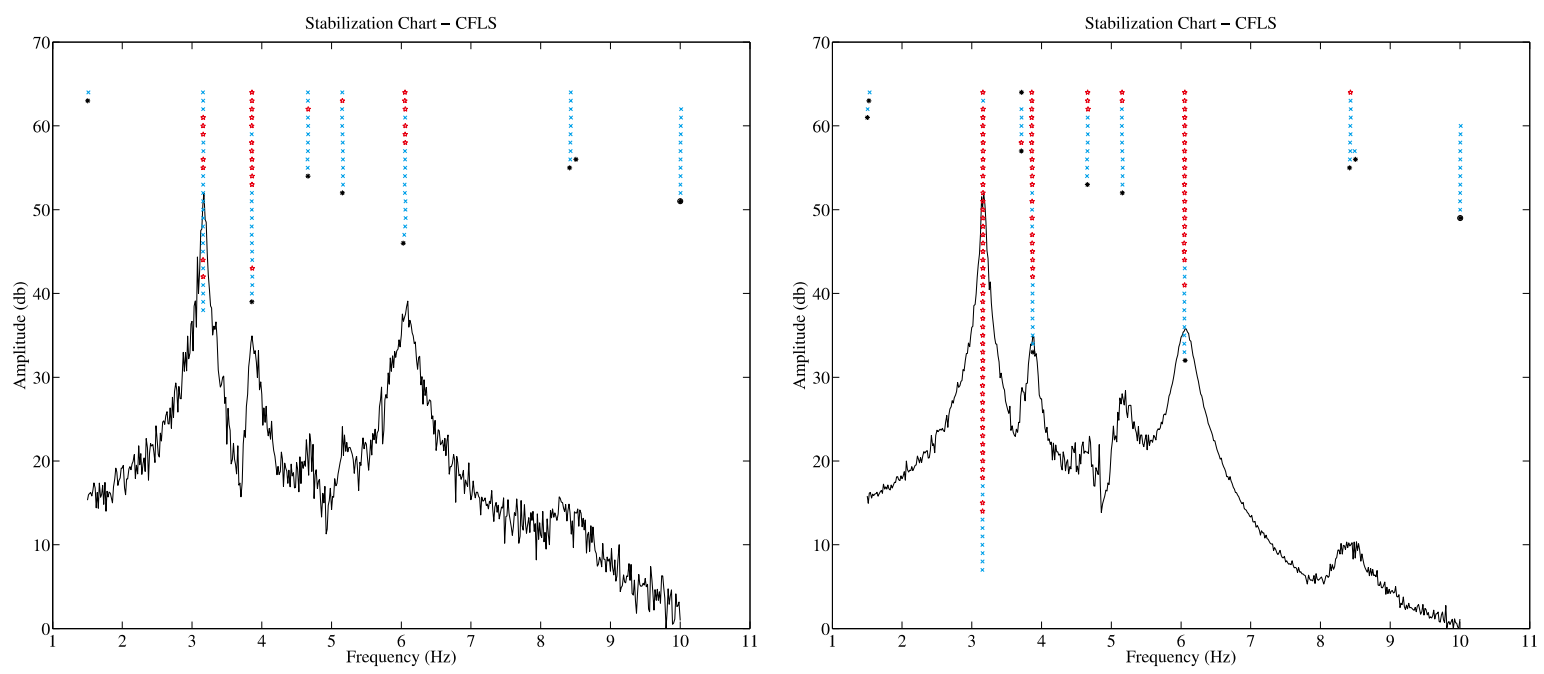

Fig. 7. Stabilization diagrams based on (left) Power Spectra OMAX (right) Transmissibility OMAX.
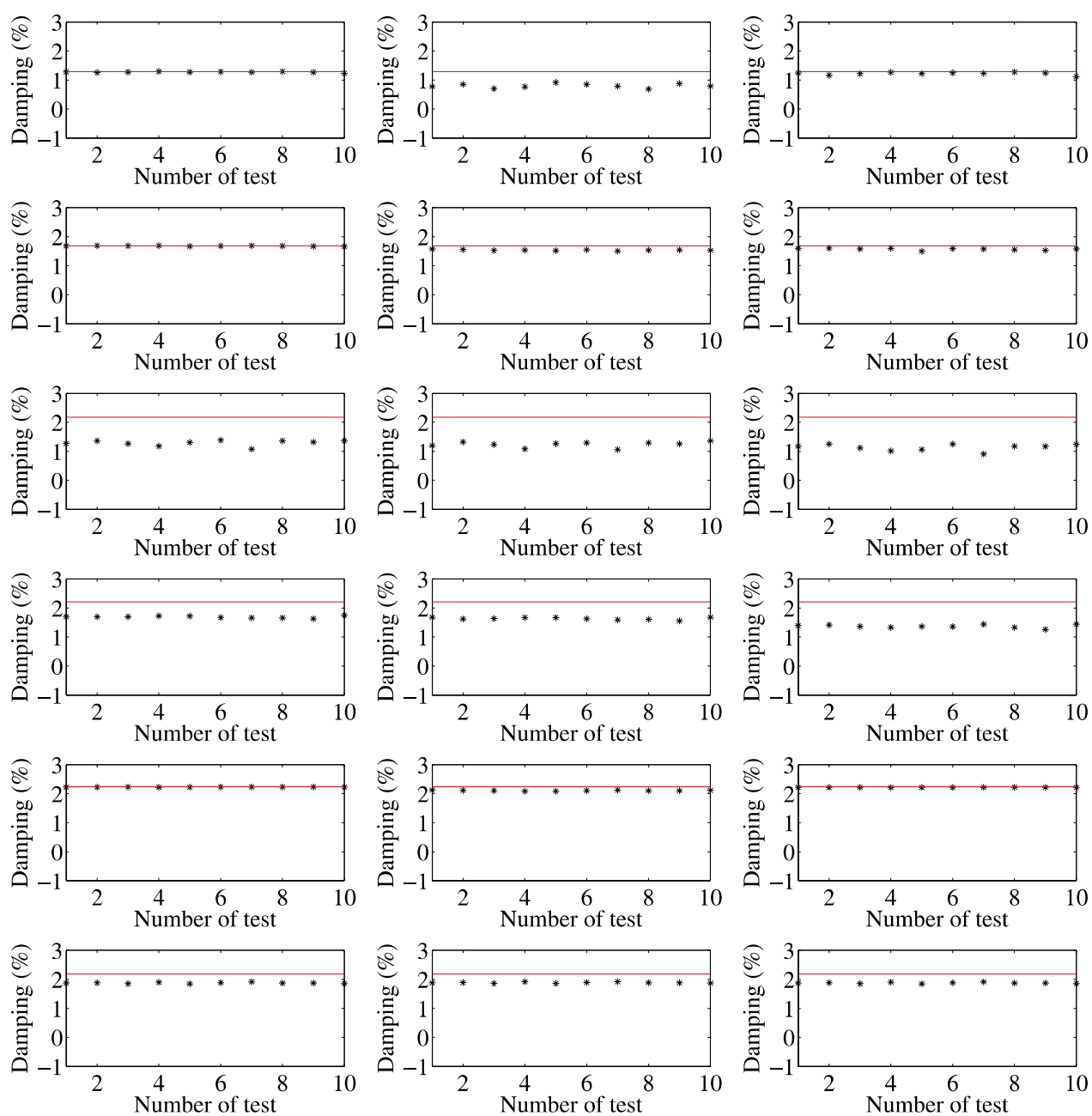

Fig. 8. Damping estimates based on (left) FRFs (middle) Power Spectra based OMAX (right) Transmissibility based OMAX. 

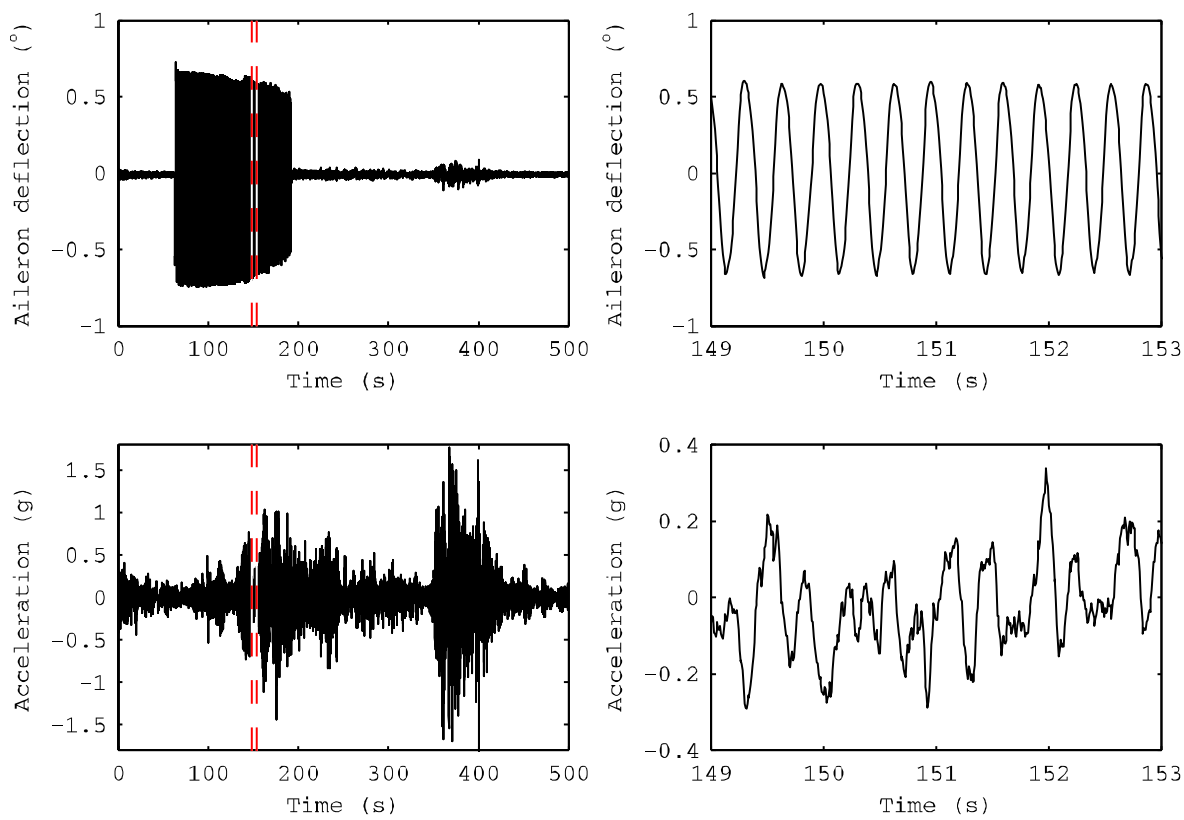

Fig. 9. Measurement of aileron deflection (top) and response acceleration at the wing (bottom), full measurement record processed (left) and detail (right).

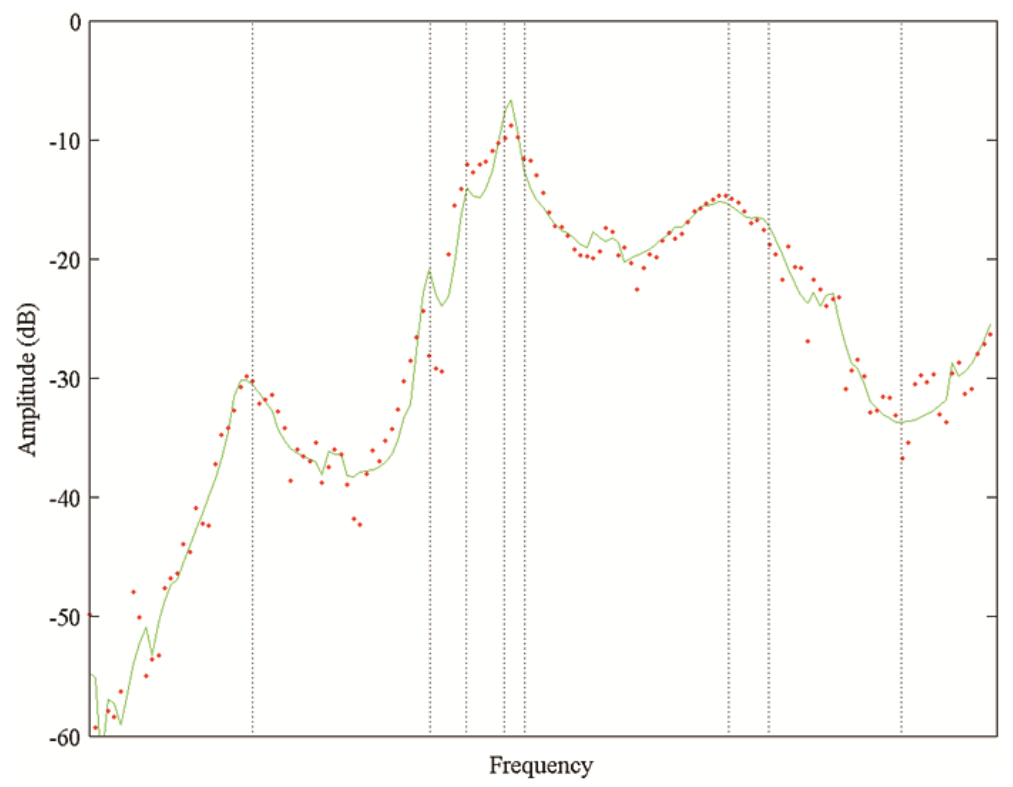

Fig. 10. Typical FRF: non-parametric estimate (red dots), OMAX FRF fit (green line); the manufacturer's identified resonant frequencies are the vertical dotted lines.

The $\mathrm{XP}^{+}$are calculated from the unexcited part using the Welch periodogram method with 11 blocks and $50 \%$ overlap, weighted with a Hanning window. Exactly the double of the number of data points is used compared with the FRF, to account for the reduction in points when calculating the positive power spectra. The $\mathrm{XP}^{+}$is also curve-fitted using a MLE with model order 32 . The transmissibility functions were obtained by comparing loading condition one (exciter on), where 7 blocks could be used, and loading condition two (turbulence only) with 11 blocks. 
First, the transmissibility approach is compared with crosspower spectrum estimate in an OMA framework. Both TM and XP are also compared to the FRF EMA approach. Figure 11 shows the relative error between the estimated resonant frequencies (left pane) and damping ratios (right pane), and the values provided by the manufacturer (which are not necessarily the true ones).
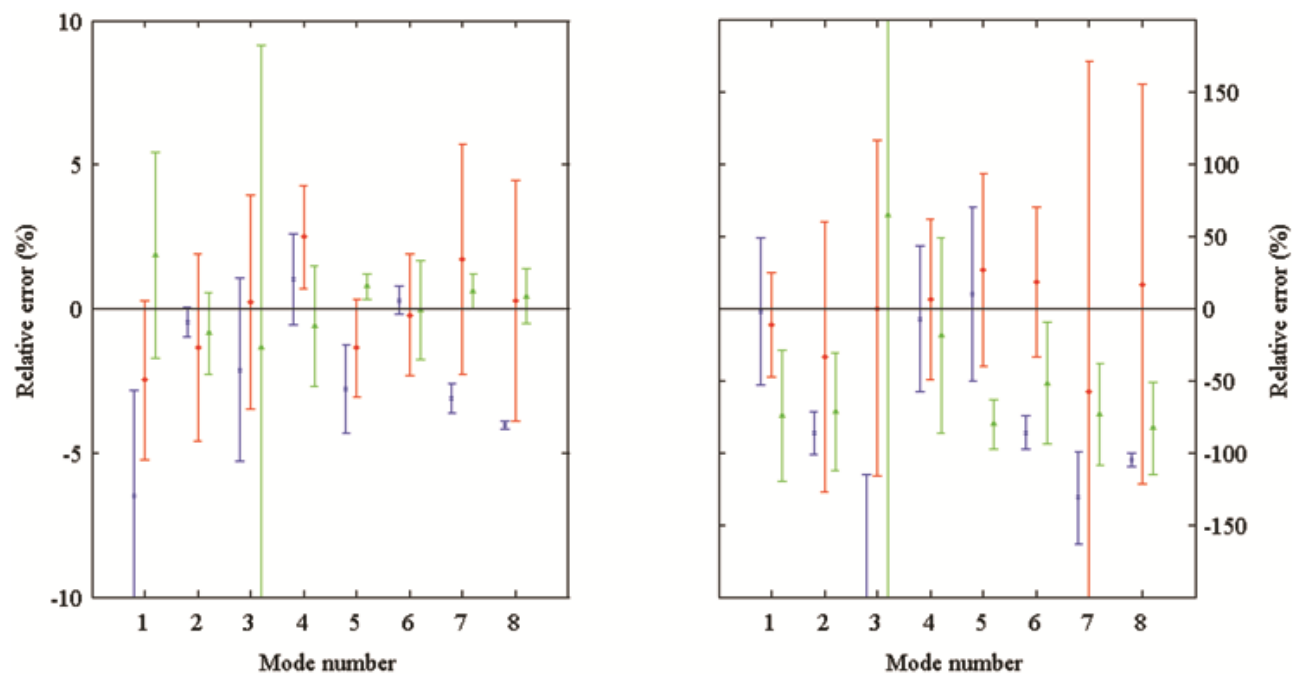

Fig. 11. Relative error of the resonant frequencies (left) and damping ratios (right) with 95\% confidence bounds, OMA TM (blue), EMA FRF (red) and $\mathrm{OMA} \mathrm{XP}^{+}$(green) based estimates.

It follows that the transmissibility OMA approach performs quite well, albeit not as good as the FRF or even the crosspower-based estimates.

Eventually both FRF and $\mathrm{XP}^{+}$, and FRF and TM, are estimated simultaneously by the same MLE estimator in the OMAX framework. Also the 95\% confidence interval on the frequency is shown. It can be verified in Fig. 12 (left pane) that the frequencies of all modes are identified well. The confidence bounds on the damping ratio estimates (right pane of Fig. 12) are larger than those on the frequencies. The transmissibility-based OMAX estimator performs quite well, comparable to the use of crosspowers, but with smaller confidence intervals for most modes.
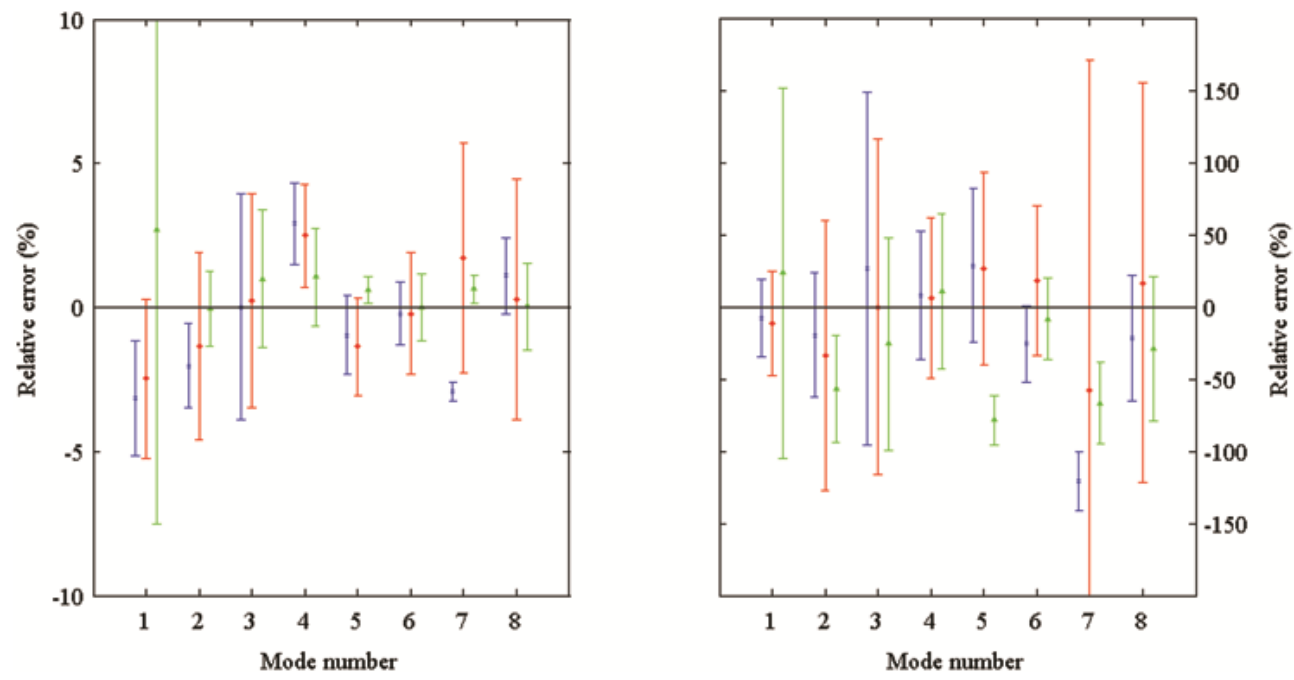

Fig. 12. Relative error of the resonant frequencies (left) and damping ratios (right) with 95\% confidence bounds, FRF + TM OMAX (blue), FRF EMA (red) and FRF $+\mathrm{XP}^{+} \mathrm{OMAX}$ (green) based estimates. 


\section{Conclusions}

In this paper, the transmissibility based output-only approach is combined with the input/output OMAX concept. This approach allows the estimation of (scaled) modal parameters in the presence of arbitrary ambient (unknown) forces and artificial (known) forces. It has been shown that FRF-data and transmissibility based data can be combined in such a way that the existing FRF-based parametric estimators can be used directly in an OMAX framework to identify the modal parameters. In our case we could observe that the OMAX approach allowed, by using a proper weighting, to improve the quality of the stabilization diagrams and the damping ratio estimates in comparison with the OMA approaches. Concluding that combining the transmissibility-based output-only approach with the input/output OMAX concept might be an idea worth pursuing and should result in a new interesting methodology in the field of operational modal analysis. This has been demonstrated by using simulations and flight flutter test data.

\section{References}

[1] M.W. Kehoe, A historical overview of flight flutter testing, Nasa technical memorandum 4720, NASA, Dryden Flight Research Center, Edwards, October, 1995.

[2] K. Deckers, T. De Troyer, E. Reynders, P. Guillaume, D. Lefeber and G. De Roeck, Applicability of low-weight pneumatic artificial muscle actuators in an OMAX framework, In Proceedings of the International Conference on Noise and Vibration Engineering ISMA, Leuven, Belgium, September, 2008, pp. 2445-2456.

[3] W. Heylen, S. Lammens and P. Sas, Modal Analysis Theory and Testing, Katholieke Universiteit Leuven, Department Werktuigkunde, Heverlee, 1997.

[4] N. Maia and J. Silva, Theoretical and Experimental Modal Analysis, Research Studies Press, Hertfordshire, 1997.

[5] R. Brincker, A. DeStefano and B. Piombo, Ambient data to analyse the dynamic behaviour of bridges: A first comparison between different techniques, In Proceedings of the 14th International Modal Analysis Conference (IMAC), Dearborn, 1996, pp. 477-482.

[6] S.R. Ibrahim, R. Brincker and J.C. Asmussen, Modal parameter identification from responses of general unknown random inputs, In Proceedings of the 14th International Modal Analysis Conference (IMAC), Dearborn, 1996, pp. 446-452.

[7] E. Parloo, Application of frequency-domain system identification techniques in the field of operational modal analysis, Ph.D., Vrije Universiteit Brussel, department MECH, Pleinlaan 2, 1050 Brussel, Belgium, 2003.

[8] B. Peeters and G. de Roeck, Reference-based stochastic subspace identification for output-only modal analysis Mechanical Systems and Signal Processing 13(6) (1999), 855-878.

[9] P. Guillaume, P. Verboven, B. Cauberghe and S. Vanlanduit, Frequency-domain system identification techniques for experimental and operational modal analysis, In Proceedings of the 13th IFAC Symposium on System Identification SYSID, Rotterdam, August, 2003.

[10] B. Cauberghe, P. Guillaume, P. Verboven and E. Parloo, Identification of modal parameters including unmeasured forces and transient effects, Journal of Sound and Vibration 265(3) (2003), 609-625.

[11] E. Reynders and G. De Roeck, Reference-based combined deterministic-stochastic subspace identification for experimental and operational modal analysis, Mechanical Systems and Signal Processing 22(3) (2008), 617-637.

[12] R. Pintelon and J. Schoukens, System Identification: A Frequency Domain Approach, IEEE Press, Piscataway, 2001.

[13] C. Devriendt and P. Guillaume, The use of transmissibility measurements in output-only modal analysis, Mechanical Systems and Signal Processing 21(7) (2007), 2689-2696.

[14] C. Devriendt, T. De Troyer, G. De Sitter and P. Guillaume, Automated operational modal analysis using transmissibility functions, In Proceedings of the International Conference on Noise and Vibration Engineering ISMALeuven, Belgium, September 2008, pp. 24572470.

[15] C. Devriendt, G. De Sitter, S. Vanlanduit and P. Guillaume, Operational modal analysis in the presence of harmonic excitations by the use of transmissibility measurements, Mechanical Systems and Signal Processing 23(3) (2009), 621-635.

[16] C. Devriendt, G. Steenackers, G. De Sitter and G. Guillaume, From operating deflection shapes towards mode shapes using transmissibility measurements, Mechanical Systems and Signal Processing 24 (2010), 665-677, 23(3) (2009), 621-635.

[17] L. Ljung, System Identification: Theory for the User, (2nd ed.), Prentice-Hall, Upper Saddle River, 1999.

[18] T. De Troyer, Frequency-domain modal analysis with aeroelastic applications, Ph.D., Vrije Universiteit Brussel, dept. MECH, Pleinlaan 2, B-1050 Brussels, Belgium, 2009.

[19] J.S. Bendat and A.G. Piersol, Engineering Applications of Correlation and Spectral Analysis, John Wiley \& Sons, New York, 1980.

[20] J. Antoni and J. Schoukens, A comprehensive study of the bias and variance of frequency-response-function measurements: Optimal window selection and overlapping strategies Automatica 43(10) (2007), 1723-1736.

[21] B. Cauberghe, Applied frequency-domain system identification in the field of experimental and operational modal analysis, $\mathrm{Ph} . \mathrm{D} .$, thesis, Department of Mechanical Engineering, Vrije Universiteit Brussel, Belgium, phd.avrg.be, 2004

[22] P. Guillaume, R. Pintelon and J. Schoukens, Description of a parametric maximum likelihood estimator in the frequency domain for multi-input, multi-output systems and its application to flight flutter analysis, Original Research Article Mechanical Systems and Signal Processing 4(5) (September 1990), 405-416. 

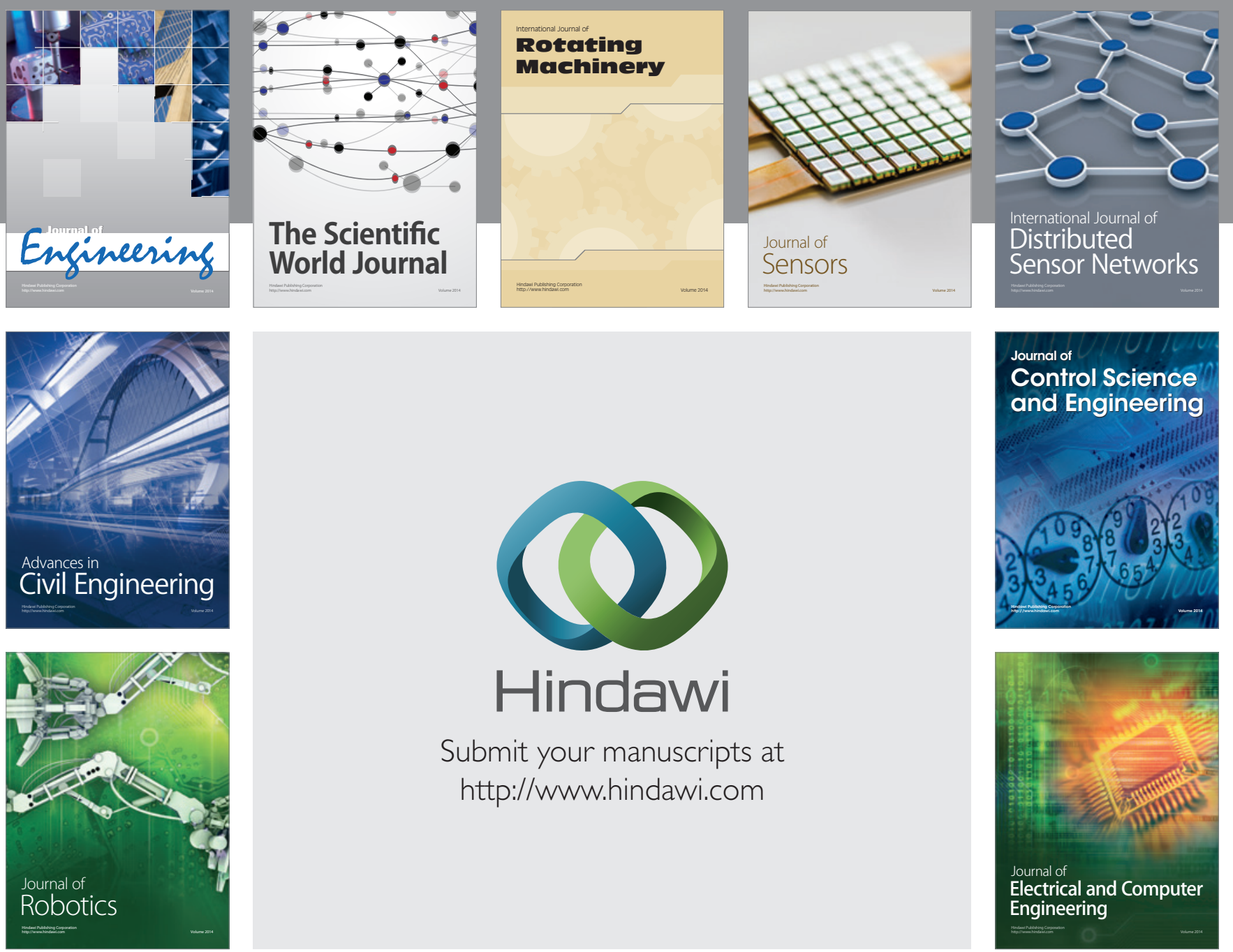

Submit your manuscripts at

http://www.hindawi.com
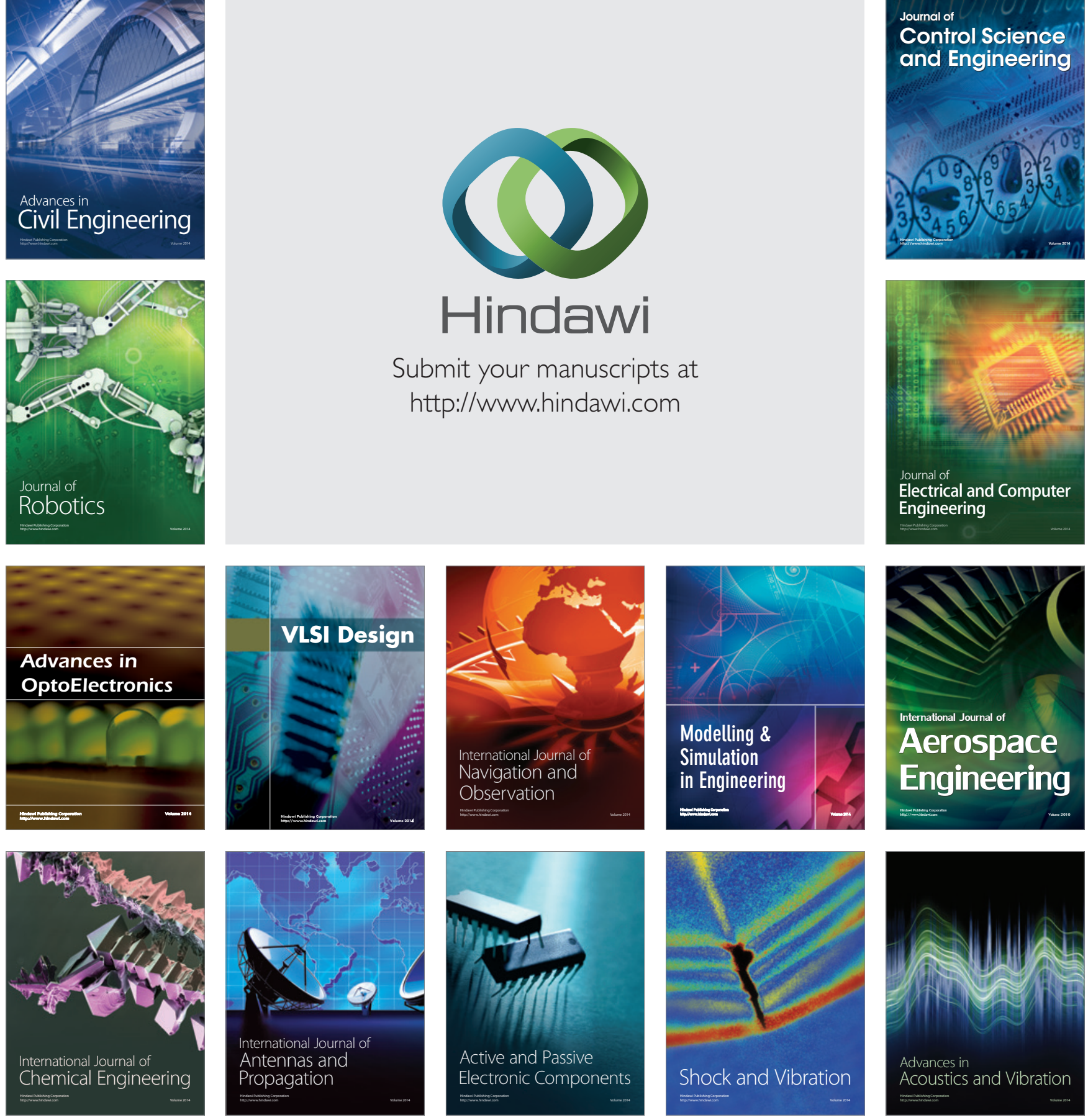